\title{
High Repetition Rate Collisional Soft X-Ray Lasers Based on Grazing Incidence Pumping
}

\author{
Bradley M. Luther, Yong Wang, Miguel A. Larotonda, David Alessi, Student Member, IEEE, \\ Mark Berrill, Student Member, IEEE, Jorge J. Rocca, Fellow, IEEE, James Dunn, R. Keenan, and \\ Vyacheslav N. Shlyaptsev
}

\begin{abstract}
We discuss the demonstration of gain-saturated high repetition rate table-top soft $X$-ray lasers producing microwatt average powers at wavelengths ranging from 13.9 to $33 \mathrm{~nm}$. The results were obtained heating a precreated plasma with a picosecond optical laser pulse impinging at grazing incidence onto a precreated plasma. This pumping geometry increases the energy deposition efficiency of the pump beam into the gain region, making it possible to saturate soft $X$-ray lasers in this wavelength range with a short pulse pump energy of only $1 \mathrm{~J}$ at $800-\mathrm{nm}$ wavelength. Results corresponding to $5-\mathrm{Hz}$ repetition rate operation of gain-saturated 14.7-nm Ni-like Pd and 32.6-nm line Ne-like Ti lasers pumped by a table-top Ti:sapphire laser are reported. We also discuss results obtained using a $1 \omega 1054-\mathrm{nm}$ prepulse and $2 \omega 527-\mathrm{nm}$ short pulse from a Nd:glass pump laser. This work demonstrates the feasibility of producing compact high average power soft $X$-ray lasers for applications.
\end{abstract}

Index Terms-Grazing incidence pumping, neon-like ions, nickel-like ions, soft $\mathrm{X}$-ray laser.

\section{INTRODUCTION}

$\mathbf{M}$ $\mathrm{UCH}$ progress has been achieved in the development of collisional X-ray lasers since their first demonstrations using fusion-class lasers as pump sources [1], [2]. Gain-saturated operation has been achieved at wavelengths as short as $5.9 \mathrm{~nm}$ [3], and their scale has been reduced from laboratorysize to table-top [4]. However, the widespread use of soft X-ray lasers in applications has been often limited by their very low repetition rate of typically one shot every several minutes, which results in very low average powers. There is significant interest

Manuscript received June 30, 2005. The work conducted at Colorado State University was supported by the NSF ERC for Extreme Ultraviolet Science and Technology under NSF Award EEC-0310717, with equipment developed under NSF Grant ECS-9 977 677. The work conducted at Lawrence Livermore National Laboratory was performed under the auspices of the U.S. Department of Energy by the University of California Lawrence Livermore National Laboratory under Contract no. W-7405-Eng-48. This work was supported in part by the W.M. Keck Foundation.

B. M. Luther, Y. Wang, M. A. Larotonda, D. Alessi, M. Berrill, and J. J. Rocca are with the National Science Foundation ERC for Extreme Ultraviolet Science and Technology, and the Electrical and Computer Engineering Department, Colorado State University, Fort Collins, CO 80523 USA (e-mail: bml@lamar.colostate.edu; wang@engr.colostate.edu; mlaroton@engr.colostate.edu; dalessi@engr.colostate.edu; berrill@engr. colostate.edu; rocca@engr.colostate.edu).

J. Dunn and R. Keenan are with the Lawrence Livermore National Laboratory, Livermore, CA 94550 USA (e-mail: dunn6@1lnl.gov).

V. N. Shlyaptsev is with the Department of Applied Science, University of California Davis-Livermore, Livermore, CA 94550 USA (e-mail: snocturne2@yahoo.com).

Digital Object Identifier 10.1109/JQE.2005.860123 in the development of gain-saturated high repetition rate soft $\mathrm{X}$-ray lasers capable of producing high average powers for a variety of studies on surfaces and materials, and for the development of unique imaging and metrology tools.

Until recently, gain-saturated operation of soft X-ray lasers at high repetition rate had been limited to wavelengths longer than $30 \mathrm{~nm}$. High average power soft X-ray laser operation was first achieved at $46.9 \mathrm{~nm}$ using capillary discharge excitation of a Ne-like Ar plasma at repetition rates up to $10 \mathrm{~Hz}$ [5]. Capillary discharge pumping allowed the utilization of small-scale high repetition rate soft $\mathrm{X}$-ray lasers in numerous applications, including the determination of optical constants by reflectometry, material ablation studies, plasma interferometry, the characterization of soft X-ray optical components [6], high resolution imaging [7], and nanopatterning [8]. Saturated operation at $10-\mathrm{Hz}$ repetition rate in the 41.8-nm line of Pd-like Xe [9], and in the 32.8-nm line of Ni-like $\mathrm{Kr}$ [10] was also reported to occur following collisional excitation in plasmas created by optical field ionization. Transient collisional electron excitation of plasmas by normal incidence irradiation of targets using a sequence of a nanosecond and a picosecond pump pulses of 3-10 J energy has produced several saturated lasers in the $12-33-\mathrm{nm}$ range. These X-ray lasers are pumped at lower repetition rates of one shot every several minutes [11], [12]. Several experiments have observed gain at $18.9 \mathrm{~nm}$ in Ni-like Mo using small pump energies, but the gain-length product obtained remained below saturation [13]-[15].

Recently, it has been demonstrated that the pump energy necessary to heat the plasmas to the temperatures required to obtain large gain can be significantly reduced by directing the picosecond pump pulse at a grazing angle of incidence to the target surface. This geometry preferentially heats a selected region of the precreated plasma with optimum density for soft X-ray amplification [16]-[22]. This pumping configuration, that is inherently traveling wave, takes advantage of the refraction of the pump beam to increase the interaction length of the rays in the gain region of the plasma [Fig. 1], thereby increasing the fraction of the pump energy absorbed in that region. Pumping of the 18.9-nm line of Ni-like Mo at $14^{\circ}$ grazing incidence with 150 $\mathrm{mJ}$ of total pumping energy from a $10-\mathrm{Hz}$ laser was reported to generate a gain-length product of $\sim 14$ [18], and the use of 1-J heating pulses at angles of incidence between $14^{\circ}$ and $23^{\circ}$ resulted in the demonstration of gain-saturated $5-\mathrm{Hz}$ repetition rate lasers in transitions of Ni-like ions [19]-[21] and Ne-like ions [22] at wavelengths as short as $13.9 \mathrm{~nm}$ for Ni-like $\mathrm{Ag}$ [21]. 

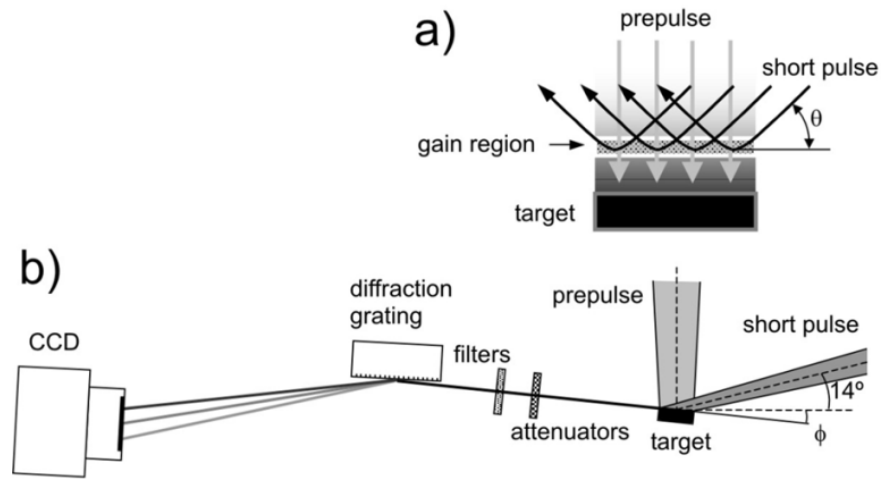

Fig. 1. (a) Schematic representation of the grazing incidence pump geometry configuration. The short pulse impinges onto the target at a grazing incidence angle $\theta$. (b) The experimental setup. The angle $\phi$ between the normal to the target surface and the axis of the prepulse beam is varied by tilting the target to obtain the different short pulse grazing incidence pump angles $\theta$ used in this work (e.g., $\phi=6$ added to the $14^{\circ}$ angle of the short pulse beam axis gives a $20^{\circ}$ grazing incidence angle).

In this paper, we discuss the demonstration of gain saturated table top collisional soft X-ray lasers pumped at grazing incidence and give examples of results of saturated laser operation obtained using two different pump wavelengths: 0.8 and 0.53 $\mu \mathrm{m}$. We present results obtained at Colorado State University (CSU) of saturated lasers in transitions of Ne-like and Ni-like ions pumped by a $5-\mathrm{Hz}$ repetition rate Ti:sapphire laser. We also discuss results obtained at Lawrence Livermore National Laboratory (LLNL) using a $2 \omega 527$-nm wavelength short pump pulse from a frequency doubled $\mathrm{Nd}$ :glass laser pump.

\section{EFFICIENT PUMPING Using GRAZING INCIDENCE GEOMETRY}

Fig. 1(a) schematically illustrates the pumping geometry used in the experiments, in which the short heating pulse impinges at grazing incidence into the precreated plasma. This grazing incidence pumping geometry (GRIP) has significant advantages over the normal incidence angle configuration conventionally used to transversely pump collisional X-ray lasers. It also overcomes the limitation in the maximum plasma length available for amplification that is associated with longitudinal pumping [13], [14].

In the case of X-ray laser plasmas heated by transverse normal incidence pumping, the majority of the energy of the heating pulse is absorbed near the critical density, where the very steep density gradients forbid the propagation of the amplified soft X-ray radiation through a sufficiently long length of the gain region. The strong refraction ejects the amplified rays off the gain medium after a distance $L_{r}$, the refraction length, that can be estimated by: $L_{r}=a \times\left(n_{\mathrm{ec}} / n_{\mathrm{e} 0}\right)^{1 / 2}$, where $n_{\mathrm{e} 0}$ is the maximum electron density, $n_{\mathrm{ec}}$ is the critical electron density at the wavelength of the amplified X-rays, and $a$ is the characteristic transverse dimension of the plasma [23]. Due to this refraction, effective amplification occurs at significantly lower densities, where the reduced density gradients allow for longer propagation lengths. However, in the normal incidence pumping geometry this plasma region is inefficiently heated because its relatively low electron density only absorbs a small fraction of the pump radiation. Typically only less than $10 \%$ of the laser pump energy is deposited in the amplification region and as a result a large laser pump energy is required to heat the plasma to the high temperatures necessary for lasing. In contrast, the grazing incidence configuration takes advantage of the refraction of the pump beam to efficiently deposit the energy of the pump beam into a region of the plasma with preselected electron density where the conditions are optimum for soft X-ray amplification. In this preselected gain region the electron density and temperature are sufficiently high for the generation of a large population inversion by transient collisional excitation, yet the density gradient is small enough to allow for effective amplification of the X-rays thorough the entire length of the gain column. A remarkable property of refraction is that this electron density, $n_{e}$, is strictly defined by only two parameters, the grazing incidence angle $\theta$ and the laser pump wavelength: $\theta=\left(n_{e} / n_{\mathrm{cp}}\right)^{1 / 2}$, where $n_{e}$ is the maximum electron density within the amplification region and $n_{\mathrm{cp}}$ is the critical density at the wavelength of the pump. Hence, when the grazing angle is changed, different parts of the density profile formed by the prepulse are preferentially heated. At a given incidence angle the pump beam is reflected at the point where it encounters the corresponding selected density $n_{e}$ [Fig. 1(a)], significantly increasing the path length of the pump beam and therefore its absorption in the gain region. Hence, a large fraction of the pump energy (typically $20 \%-50 \%$ ) can be selectively deposited into the gain region. In addition, the grazing incidence pumping configuration has the advantage of an intrinsically traveling wave for a range of incidence angles of interest, a fact that simplifies the experimental set up. The optimization of amplification along the entire length of the medium requires that: a) the refraction of the soft X-ray laser radiation is minimized, and $\mathrm{b}$ ) the mismatch of traveling wave pump speed and photon speed is kept small compared to the gain lifetime. When the initial grazing angle is small, e.g., $\theta<20^{\circ}$, this mismatch $\sim(L / c) \times[1-\cos (\theta)]$ is also negligibly small. However, for larger angles it could reach substantial values. For example with a plasma column length of $L=1 \mathrm{~cm}$ and $\theta=30^{\circ}$ the mismatch is $9 \mathrm{ps}$, which could be comparable to transient gain lifetime which can be in the range 3-15 ps depending on the maximum electron density achieved (determined by the grazing angle) and the pump laser duration.

The above discussion is illustrated in Fig. 2 by model simulation results for the specific case of the 14.7-nm Ni-like Pd laser for which we present experimental results in the following section. These results were obtained using a $1.5 \mathrm{D}$ hydrodynamic/atomic physics code with multicell radiation transport and beam refraction developed at CSU. ${ }^{1}$ The simulation compares normal incidence and $20^{\circ}$ grazing incidence irradiation of a 4-mm-long Pd plasma heated by a 1-J pulse of 8-ps duration focused to an intensity of $8 \times 10^{13} \mathrm{~W} \cdot \mathrm{cm}^{-2}$. The plasma is assumed to be created by a $350-\mathrm{mJ}$ prepulse of 120 -ps duration impinging at normal incidence onto the target. In the case of normal incidence pumping the picosecond pulse rapidly heats the region near the critical density, generating a transient gain

${ }^{1}$ Colorado State University Hydrodynamic/Atomic Physics Code Developed by M. Berrill and J. J. Rocca 

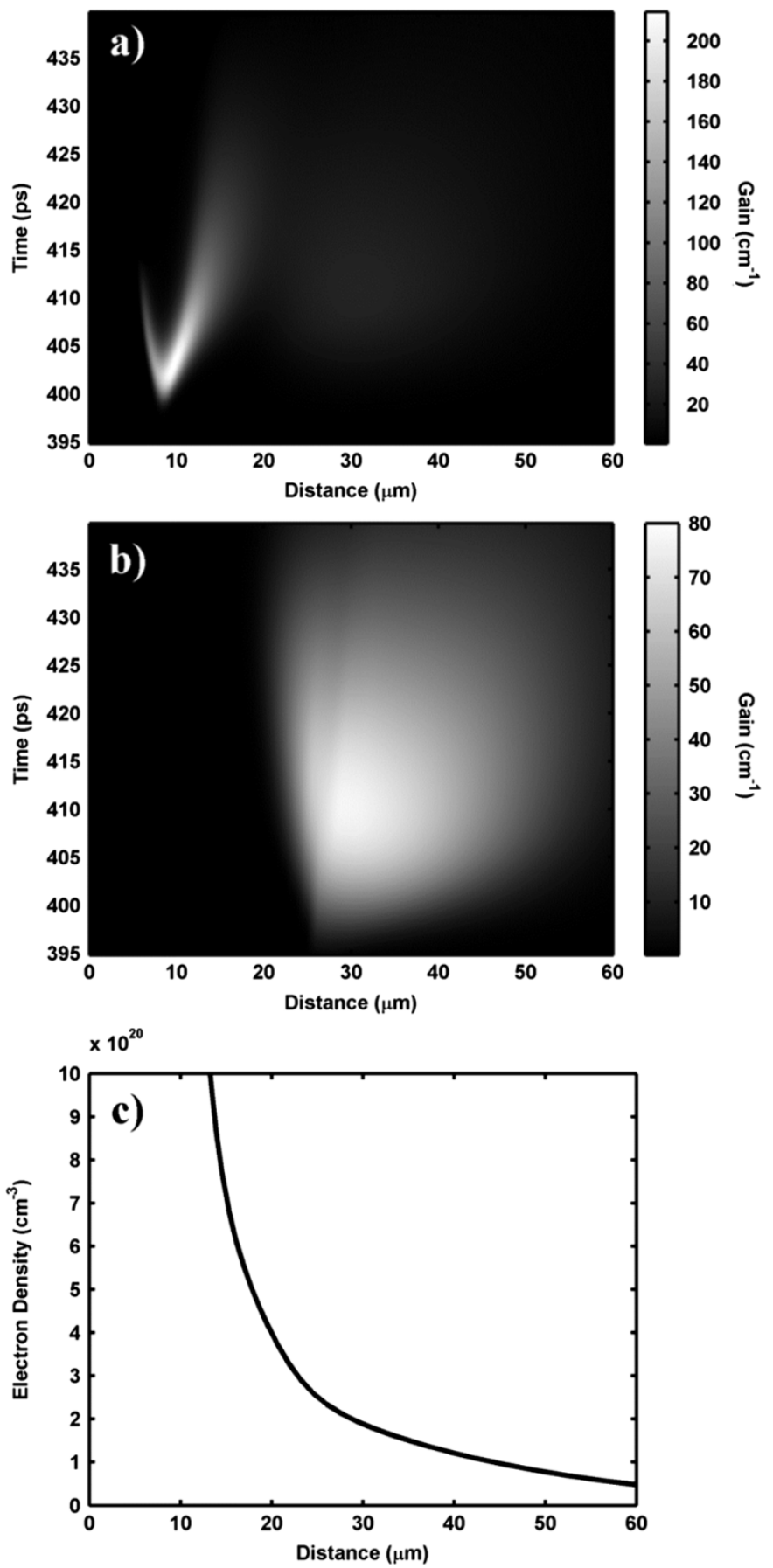

Fig. 2. Simulated gain maps for a Ni-like Pd 14.7-nm laser at the moment of peak gain for (a) normal incidence irradiation, (b) $20^{\circ}$ grazing incidence irradiation, and (c) simulated electron density profile of the prepulse at the moment of the short pulse arrival. At normal incidence the region of large gain coefficient coincides with large density gradients that rapidly refract the beam out of the narrow gain region. For a pump angle of $20^{\circ}$ the beam heats the plasma where $N_{e}=2 \times 10^{20} \mathrm{~cm}^{-3}$, creating large gain in a region where reduced refraction allows the beam to effectively propagate and amplify. The plasma is assumed to be created by a 120 -ps normal incidence prepulse with an intensity of $2.4 \times 10^{12} \mathrm{~W} \cdot \mathrm{cm}^{-2}$, and heated after a 300-ps delay by an 8-ps duration, 800-nm wavelength, pulse focused to an intensity of $8 \times 10^{13} \mathrm{~W}$. $\mathrm{cm}^{-2}$, both line foci are $30-\mu \mathrm{m}$-wide FWHM.

with a peak gain coefficient of about $200 \mathrm{~cm}^{-1}$ at a distance of about $10 \mu \mathrm{m}$ from the target, where the value of the electron density is close to $2 \times 10^{21} \mathrm{~cm}^{-3}$. However in this region the amplified soft $\mathrm{X}$-rays refract out of the high gain region in only several hundred micrometers, inhibiting amplification to large intensities. In contrast, at $20^{\circ}$ grazing incidence angle the pump beam energy is coupled into the region of the plasma with $n_{e}=2 \times 10^{20} \mathrm{~cm}^{-3}$, where the peak small signal gain is computed to be $80 \mathrm{~cm}^{-1}$ and $L_{r}$ is larger than the 4-mm target length, allowing amplification to intensities that exceed the gain saturation intensity. The simulations show that for the case of the grazing incidence pumped lasers discussed below the fraction of the pump energy deposited into the gain region is of the order of $20 \%-30 \%$, significantly greater than the $5 \%-8 \%$ corresponding to the normal incidence pumping case. This significantly reduces the amount of pump energy required for lasing in the gain-saturated regime, making possible the development of high repetition rate table-top lasers.

\section{Demonstration OF High-REPETITION-RATE OPERATION OF SATURATED Ni-LiKE Pd AND Ne-LiKe Ti LASERS PUMPED WITH A TABLE-TOP Ti:SAPPHIRE LASER}

In this section, we discuss experiments conducted at CSU that achieved saturated laser operation in lines of several ions at wavelengths between 13.9 and $32.6 \mathrm{~nm}$ at $5-\mathrm{Hz}$ repetition rate. The experiments made use of a Ti:sapphire pump laser system that generates picosecond duration heating pulses of $\sim 1 \mathrm{~J}$ energy. Saturated laser operation was achieved in the $4 d^{1} S_{0} \rightarrow$ $4 p^{1} P_{1}$ transition of Ni-like ions at wavelengths ranging from $18.9 \mathrm{~nm}$ (Ni-like Mo) [19], [20] to $13.9 \mathrm{~nm} \mathrm{Ni-like} \mathrm{Ag} \mathrm{[21],} \mathrm{and}$ in the $3 p^{1} S_{0} \rightarrow 3 s^{1} P_{1}$ transitions of Ne-like Ti and Ne-like V at 32.6 and $30.4 \mathrm{~nm}$, respectively. In what follows, results corresponding to lasers in Ni-like $\operatorname{Pd}(Z=46)$ at $14.7-n m$ wavelength and on Ne-like $\mathrm{Ti}(Z=22)$ at 32.6- and 30.1-nm wavelength are discussed in detail.

\section{A. Experimental Setup: Optimization of Time Delay and Angle of Incidence of the Picosecond Pump Pulse}

The $\lambda=800$-nm pump laser used in these experiments consists of a mode-locked Ti:sapphire oscillator and three stages of chirped-pulse amplification. Nanojoule pulses from a Kerr mode-locked laser oscillator are stretched to about 180 ps and subsequently amplified in a chain of three Ti:sapphire amplifiers. Eight passes through the first stage amplifier increases the pulse energy to about $2 \mathrm{~mJ}$, while the pulse width narrows to about 120 ps. Further amplification in a five-pass second stage bow-tie amplifier further increases the pulse energy to about $200 \mathrm{~mJ}$. The output of the second stage amplifier is spatially filtered and injected into a final three-pass amplifier that is pumped by a 5-J frequency doubled Nd:YAG laser. This final amplification stage increases the $\lambda=800-\mathrm{nm}$ laser pulse energy up to about $2 \mathrm{~J}$. A multilayer coated beam splitter was placed at the output of the third stage amplifier to direct $20 \%$ of the uncompressed 120-ps duration laser pulse energy to the prepulse arm. The rest of the third stage output is sent to a vacuum grating compressor, where it is compressed to 8 ps. Compressed pulses of $1 \mathrm{~J}$ energy are routinely obtained. The laser can operate at a repetition rate of $10 \mathrm{~Hz}$ corresponding to the repetition frequency of the two Nd:YAG lasers used to pump the amplifiers. However, for all the soft X-ray laser experiments conducted to date the repetition rate of the third stage amplifier was reduced to $5 \mathrm{~Hz}$ to improve the pump beam mode quality. 
The pumping geometry used to obtain soft X-ray lasing in Ne-like and Ni-like ions is schematically illustrated in Fig. 1(b). The targets consisted of 4-mm-wide polished slabs that had a thickness of 1 or $2 \mathrm{~mm}$ depending on the material of interest. Plasmas were formed by irradiating the targets at near normal incidence with a prepulse with an energy of $0.35 \mathrm{~J}$, that was preceded by a $10-\mathrm{mJ}$ prepulse about $5 \mathrm{~ns}$ before. The prepulse was focused into a 4.1-mm-long $\times 30-\mu \mathrm{m}$-wide full-width at half-maximum (FWHM) line using the combination of an $f=$ $67.5 \mathrm{~cm}$ spherical lens and an $f=200 \mathrm{~cm}$ cylindrical lens. The plasma created by this irradiation was allowed to expand for a chosen time delay and was then rapidly heated by the 8-ps FWHM duration pulse impinging at a selected grazing incidence angle. The picosecond heating pulse was also focused into a $30-\mu$ m-wide FWHM line using a $f=76.2 \mathrm{~cm}$ focal length multilayer-coated parabolic mirror placed at $7^{\circ}$ from normal incidence. The off axis placement of the paraboloid formed an astigmatic focus that resulted in a line that was further elongated to $4.1 \mathrm{~mm}$ when intercepted at grazing incidence by the target. The overlap of the two line foci on target was monitored and adjusted by imaging the target with a $f=25 \mathrm{~cm}$ lens onto a charge coupled device (CCD).

The plasma emission was analyzed and recorded using a flat field spectrograph composed of a $1200 \mathrm{l} / \mathrm{mm}$ gold-coated variably spaced ruling spherical grating, placed at $87^{\circ}$ and a $2048 \times 2048$ pixel, 1 in $^{2}$, back-illuminated CCD detector array placed in the image plane of the grating located at 48 $\mathrm{cm}$ from the target. The plasma radiation was partially filtered with thin film filters of $\mathrm{Al}$ or $\mathrm{Zr}$ in the Ne-like $\mathrm{Ti}$ and Ni-like Pd laser experiments, respectively. A set of metallic meshes of measured transmissivity were positioned between the target and the grating to further attenuate the beam with the purpose of avoiding saturation of the CCD detector by the intense soft $\mathrm{X}$-ray laser beams. When more than one mesh was used care was taken to avoid the formation of Moiré patterns that can cause large spatial variations of the transmissivity.

The surface of the target was tilted by an angle $\phi$ to form a selected grazing incidence angle $\theta$ ranging from $14^{\circ}$ to $26^{\circ}$ with respect to the axis of the short pulse beam [see Fig. 1(b)]. In each experiment, this incidence angle $\theta$ was optimized to maximize the energy deposition into the gain region to allow for maximum soft X-ray laser output power. If the incidence angle is too small, the pump beam refraction occurs in a plasma region where the electron density and hence the gain is low. On the contrary, if the incidence angle is selected to be excessively large, the pump beam penetrates deeper in the plasma depositing its energy on a higher density region closer to the target surface where the electron density gradients are too steep for optimum amplification. Another sensitive variable on these experiments is the time delay between the prepulse and the short grazing incidence pulse. Fig. 3 shows how the optimum delay between pump pulses for lasing in the $4 d \rightarrow 4 p$ transition of Ni-like ions decreases as $Z$ is increased. The optimum delay drops from 700 ps for Ni-like $\operatorname{Mo}(Z=42)$ [19], [20] to $100 \mathrm{ps}$ for $\operatorname{Sn}(Z=50)$ [21]. This is consistent with the fact that the high degrees of ionization necessary for lasing in higher $Z$ ions are reached only in the early stages of the prepulse plasma expansion. Therefore, for lasing in higher $Z$ ions the optimum delay that results from the tradeoff between increased Ni-like ion fraction for maximum gain coefficient and decreased electron density gradients for re-

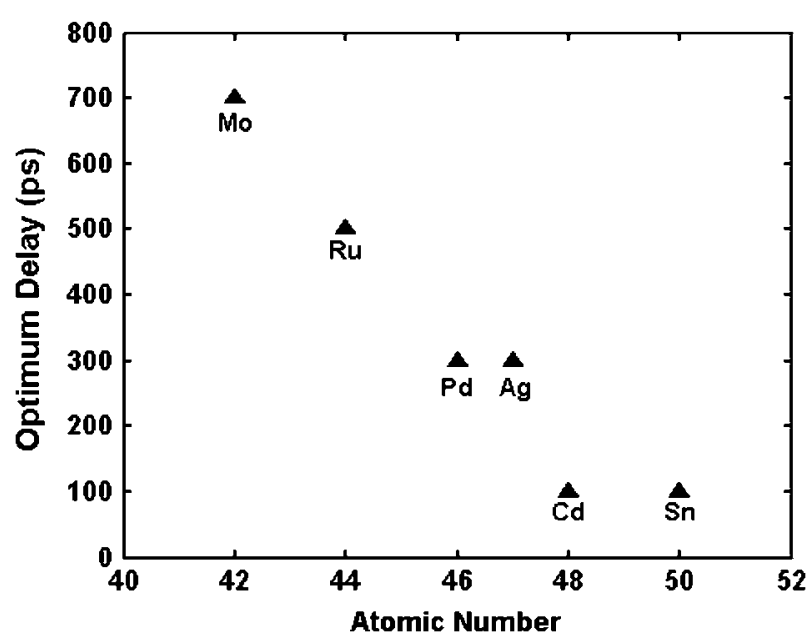

Fig. 3. Optimum delay between the $120-\mathrm{ps}, \sim 350-\mathrm{mJ}$ prepulse, and the 8-ps, $\sim 1$-J short pulse, for $4 d^{1} S_{0} \rightarrow 4 p^{1} P_{1}$ lasers on the different Ni-like ions; $Z=42$ (Mo), $Z=44(\mathrm{Ru}), Z=46(\mathrm{Pd}), Z=47$ (Ag), $Z=48(\mathrm{Cd})$, and $Z=50(\mathrm{Sn})$
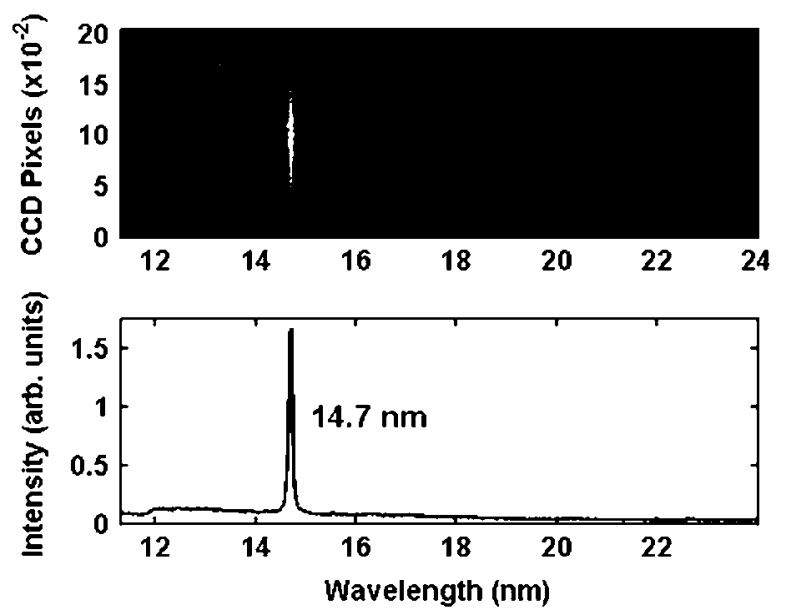

Fig. 4. Single shot on axis spectra of 4-mm-long line focus Pd plasma showing lasing in the 14.7-nm transition.

duced refraction loss occurs at shorter delays. In contrast, the incidence angle that maximizes the soft X-ray laser output intensity increases with $Z$, since a deeper pump beam penetration in the plasma is needed to reach the higher electron densities that are optimum for efficient pumping of the shorter wavelength transitions in the higher $Z$ ions. The angle for maximum soft $X$-ray laser output energy was measured to increase from $20^{\circ}$ for the 18.9-nm line of Ni-like Mo [20] to $23^{\circ}$ for the 11.9-nm line of Ni-like Sn [21]. At these angles refraction couples the energy of the short pulse laser pump energy into plasma regions having an electron density of $2 \times 10^{20} \mathrm{~cm}^{-3}$ and $2.8 \times 10^{20}$ $\mathrm{cm}^{-3}$, respectively.

\section{B. Characteristics of a 5-Hz Repetition Rate 14.7-nm Ni-Like Pd Laser}

Fig. 4 shows an on axis spectra corresponding to plasmas generated irradiating 4-mm-long targets of Pd with a $\sim 350 \mathrm{~mJ}$ main prepulse followed 520 ps later by $\sim 1-\mathrm{J}, 8$-ps short pulse impinging at a grazing incidence angle of $20^{\circ}$. Strong lasing is observed in the $4 d^{1} S_{0} \rightarrow 4 p^{1} P_{1}$ line of the Ni-like Pd at $14.7 \mathrm{~nm}$. The divergence in the direction perpendicular to the 


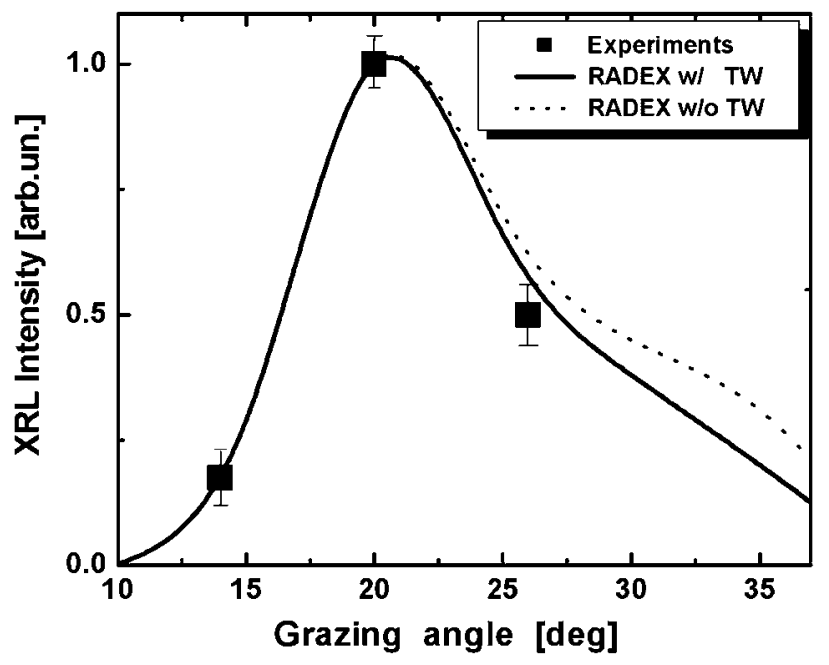

Fig. 5. Measured dependence of laser output intensity of the 14.7-nm laser line of Ni-like Pd as a function of grazing incidence angle. The two curves represent results of RADEX simulations for the cases with and without the traveling wave mismatch effect included.

target was measured to be $\sim 10$ milliradians. At this angle of incidence refraction couples the pump light into a plasma region in which the electron density is $2 \times 10^{20} \mathrm{~cm}^{-3}$. Fig. 5 shows the measured dependence of the laser output intensity as a function of grazing incidence angle. Angles of $14^{\circ}$ and $26^{\circ}$ showed a significantly lower soft X-ray laser output intensity. The results are in good agreement with simulations conducted using the code RADEX. The model includes one-dimensional (1-D) expansion of laser produced plasma under transverse prepulse and grazing incidence main pulse, atomic kinetics, the refraction of the main pump pulse, and refraction of X-ray radiation pulse and its amplification computed taking into account the saturation of amplified spontaneous emission (ASE) signal. The calculations show that optimum grazing incidence angle for $\mathrm{Pd}$ should occur at $\sim 20^{\circ}-22^{\circ}$, which is in very good agreement with the experimental data. Since the maximum heated density rapidly increases with angle as $\sim n_{e}^{2}$, the gain coefficient grows from 40 to about $100 \mathrm{~cm}^{-1}$ when angle changes from $15^{\circ}$ to $40^{\circ}$. The gain maximum is typically reached near the turning point. With increasing grazing angle the temperature increases in the turning point but decreases away from it. As a result of the drop in the temperature in most of the gain volume, and of the larger soft X-ray laser beam refraction that accompanies the increase in density, the laser intensity eventually stops any further growth and ultimately decreases for large angles. The optimum angle is therefore reached when the gain is high and density gradients still allow the soft $\mathrm{X}$-ray radiation to be amplified thought the entire $4 \mathrm{~mm}$ length of the gain volume. The calculations also allow us to distinguish the influence of the mismatch between the traveling wave of the pump and the soft X-ray laser pulse from other factors that affect the laser output intensity. The results show that for the 4-mm-long targets used in the experiments, the traveling wave pump-to-time of flight mismatch does not exceed $\sim 2$ ps even at large grazing angles of $30^{\circ}-40$, respectively. In our particular case corresponding to computed gain duration of 6-8 ps, this mismatch is generally negligible. Hence, the observed decrease of X-ray laser output at large angles is associated mostly with refraction that deflects the ampli-

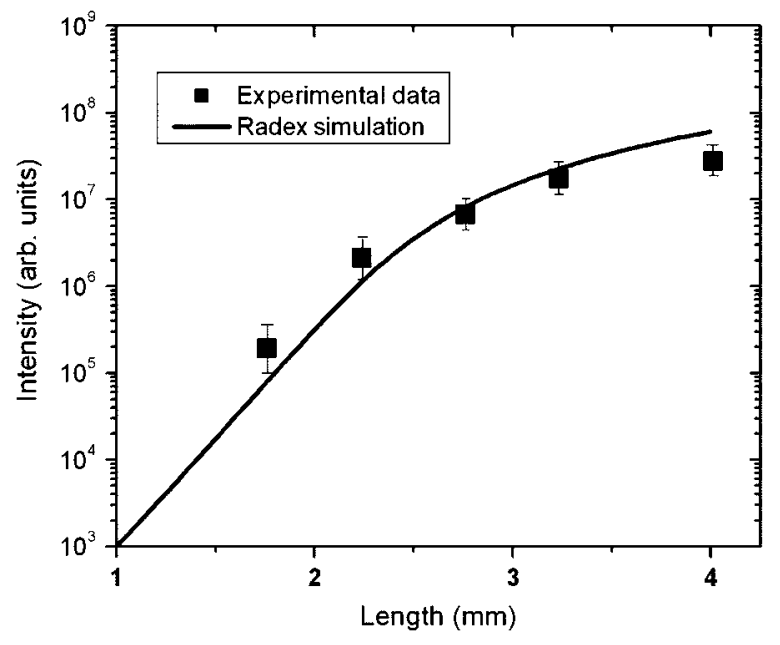

Fig. 6. Intensity versus length for the 14.7-nm line of Ni-like Pd. Each of the points is an average of ten or more laser shots. The line is the result of a simulation preformed with the code RADEX corresponding to a small signal gain coefficient is $63 \mathrm{~cm}^{-1}$ and the gain-length product is $g \times l=17.2$.

fied beam into plasma density region of lower density and lower temperature and consequently lower gain.

In order to measure gain, the variation of the laser line intensity as a function of plasma length was recorded using targets with steps ranging from 1.5 to $4 \mathrm{~mm}$ in length. Fig. 6 shows a gain measurement for the 14.7-nm line of $\mathrm{Ni}$-like $\mathrm{Pd}$, in a plasma heated with $1 \mathrm{~J}$ of short pulse arriving at $20^{\circ}$ grazing incidence with a delay of 520 ps. The intensity is observed to increase rapidly as a function of target length, until it rolls off into saturation. The line in Fig. 6 is the result of a simulation performed with the code RADEX, that constitutes a good fit to the experimental data. The small signal gain coefficient of this simulated curve is $63 \mathrm{~cm}^{-1}$ and the gain-length product is $g \times l=17.2$, a value that is compatible with saturation of the gain in collisional X-ray laser systems. A fit of the data with the gain expression by Tallents et al., that also takes into account the effect of gain saturation [24], results in a slightly smaller gain-length product of $g \times l=16.1$. Using the same set up a very similar gain-length product, 16.8, was measured for the 13.9-nm line of Ni-like Ag [21]. The 14.7-nm soft X-ray laser pulse energy was estimated from the counts on the CCD taking into account the quantum efficiency of the detector and the losses. The most intense shots obtained with a 4-mm target are estimated to exceed $0.6 \mu \mathrm{J}$. Assuming the laser pulse width is that predicted by the RADEX model computations, $\sim 5 \mathrm{ps,}$ and an exit beam diameter corresponding to the width of the pump beam, $30 \mu \mathrm{m}$, this pulse energy corresponds to a laser intensity of about $1.8 \times 10^{10}$ $\mathrm{W} \cdot \mathrm{cm}^{-2}$, which exceeds the computed saturation intensity of $3-7 \times 10^{9} \mathrm{~W} \cdot \mathrm{cm}^{-2}$ of these lines at the plasma conditions corresponding to $20^{\circ}$ grazing angle pumping.

Fig. 7 shows the variation of the intensity of the $14.7-\mathrm{nm}$ line as a function of delay time between the prepulse and the short pulse. Strong lasing is observed to occur over a broad range of time delays, ranging from about 100 to 600 ps. Fig. 8 demonstrates $5-\mathrm{Hz}$ repetition rate operation of the Ni-like Pd laser for 200 consecutive shots. The data was obtained by binning the individual pixels of the CCD detector to allow for read-out of the 


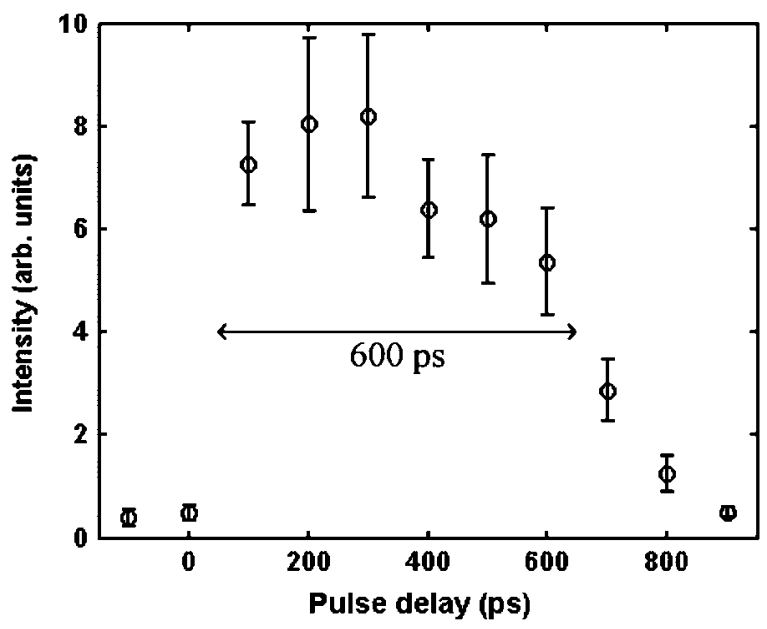

Fig. 7. Intensity versus time delay between the 120-ps main prepulse and the 8-ps grazing incidence short pulse for the 14.7-nm laser line of Ni-like Pd.

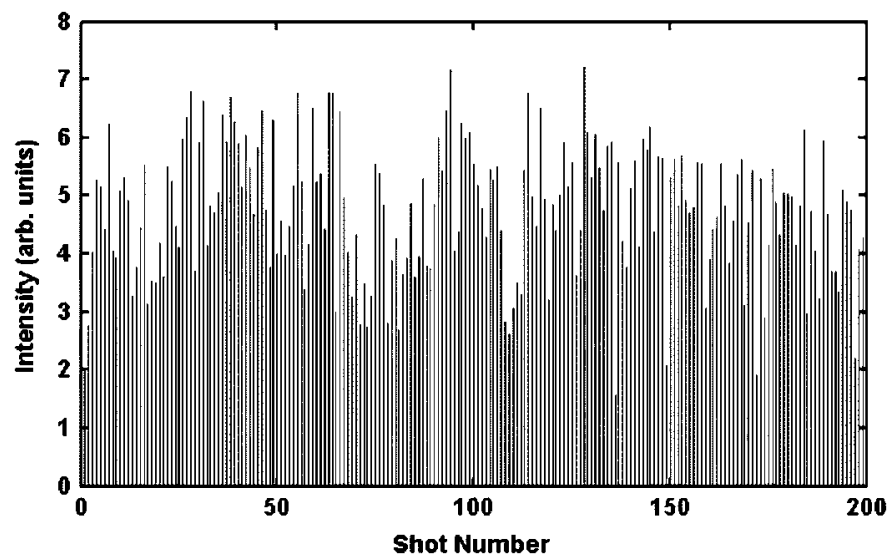

Fig. 8. Shot to shot variation of the intensity of the 14.7-nm laser line of Ni-like $\mathrm{Pd}$ for a series of 200 laser shots obtained at $5-\mathrm{Hz}$ repetition rate with $\sim 1 \mathrm{~J}$ of short pulse excitation. The stability is characterized by a standard deviation that is $24 \%$ of the mean.

array at high repetition rates while moving the target at a velocity of $0.2 \mathrm{~mm} / \mathrm{s}$. Lasing is observed for all pump laser shots. Analysis of the data shows the shot to shot intensity variation is characterized by a standard deviation of $\sim 30 \%$ of the mean. The average pulse energy is estimated to be $\sim 400 \mathrm{~nJ}$, which amounts to an average power of $2 \mu \mathrm{W}$.

\section{Characteristics of a 5-Hz Repetition Rate Ne-Like Titanium Laser}

In the case of the Ne-like $\mathrm{Ti}$, lasing was obtained in two laser lines: the $3 p^{1} S_{0} \rightarrow 3 s^{1} P_{1}$ transitions at $32.6 \mathrm{~nm}$, and the $3 d^{1} P_{1} \rightarrow 3 p^{1} P_{1} 30.1-\mathrm{nm}$ line which relies on strong re-absorption of the $2.335-\mathrm{nm}$ transition linking the $3 d^{1} P_{1}$ laser upper level to the ion ground state for inversion [25]. The 30.1-nm line is most intense at shorter delays between 400 and $500 \mathrm{ps}$ and for larger grazing incidence angles, both conditions corresponding to a larger density of Ne-like ground state ions which increase the trapping of the $2.335-\mathrm{nm}$ line.

Fig. 9 shows the measured variation of the intensity of the two Ti laser lines as a function of grazing incidence angle. At an incidence angle of $17^{\circ}$ strong lasing was observed only for the $3 p \rightarrow 3 s$ line, at $32.6 \mathrm{~nm}$. The output intensity increased

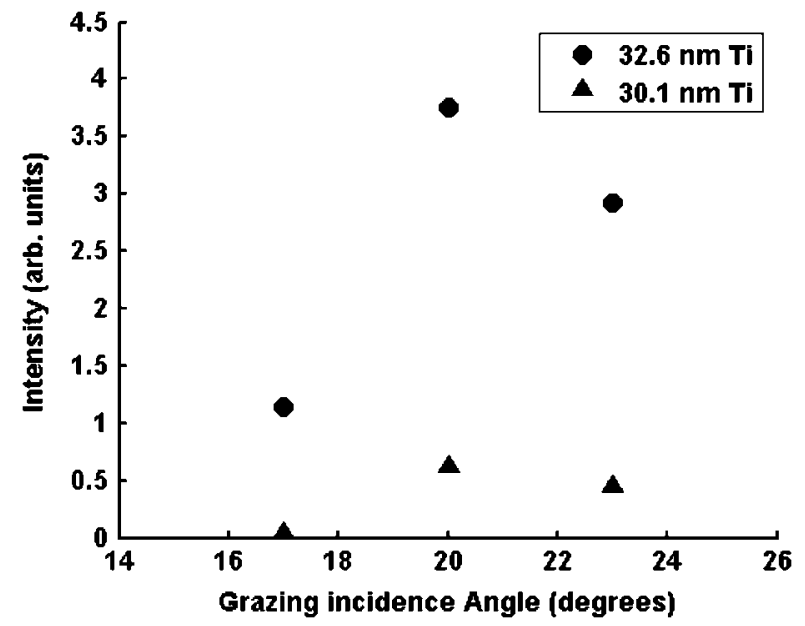

Fig. 9. Laser output intensity corresponding to the 30.1- and 32.6-nm lines of Ne-like Ti versus grazing incidence angle of the 8-ps duration heating pulse.

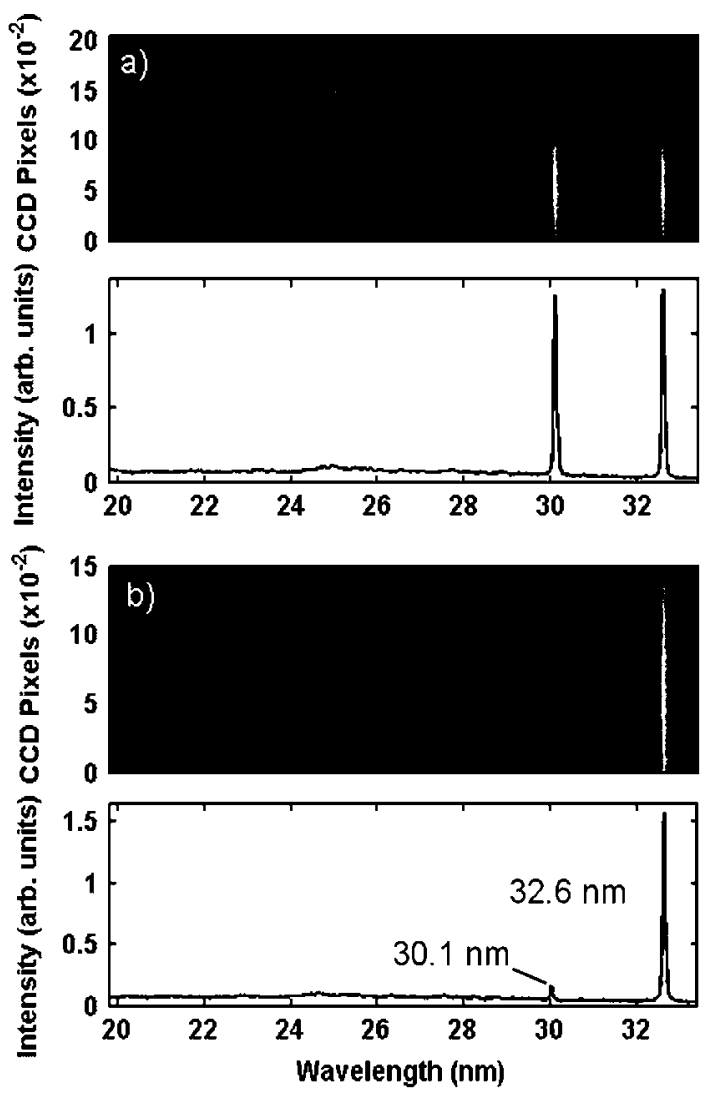

Fig. 10. On-axis Ne-like Ti spectra, measured at $20^{\circ}$ grazing incidence. (a) 520-ps delay between prepulse and short pulse, both lines show similar intensity. (b) 620-ps delay, where the 32.6-nm dominates the spectrum.

significantly for an angle of $20^{\circ}$, and decreased when the angle was further increased to $23^{\circ}$. Lasing in the $30.1-\mathrm{nm} 3 d \rightarrow 3 p$ line was observed to be strong for $20^{\circ}$ and $23^{\circ}$ grazing incidence. Two spectra for different delays between pump pulses are shown on Fig. 10. Fig. 10(a) corresponds to a delay of 520 ps and shows strong lasing for both lines. Fig. 10(b) which corresponds to a longer delay of $620 \mathrm{ps}$, is completely dominated by the 32.6-nm line. Fig. 11 illustrates the output of the Ne-like Ti laser at 32.6 and at $30.1 \mathrm{~nm}$ as a function of the delay between pump pulses for a grazing incidence angle of $20^{\circ}$. The 


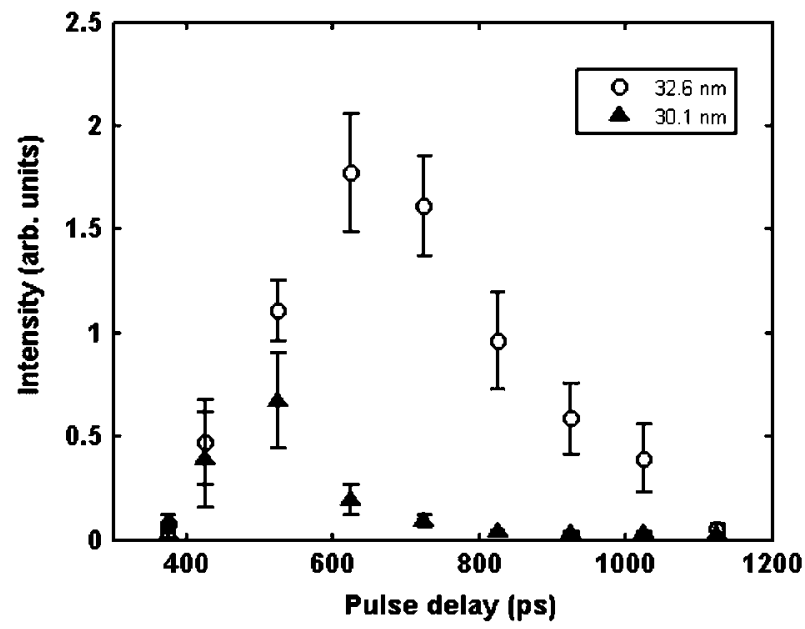

Fig. 11. Variation of the intensity of the 32.6- and 30.1-nm lines of Ne-like $\mathrm{Ti}$ as a function of time delay between the 120 -ps prepulse and the 8 ps. The grazing incidence angle was $20^{\circ}$.

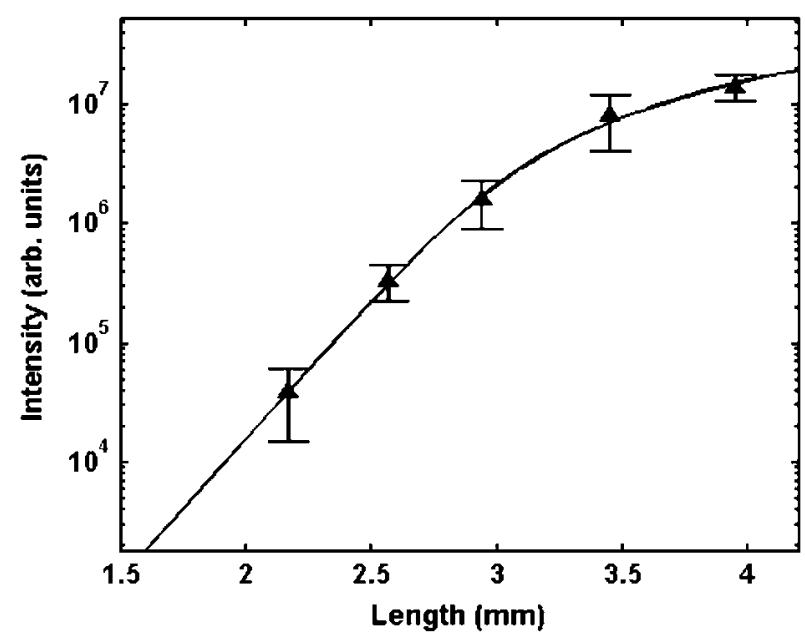

Fig. 12. Laser output intensity of the 32.6-nm line of Ne-like Ti as a function of target length. A best fit of the data with an analytical expression that relates the gain and the output laser intensity taking into account gain saturation yields a small signal gain coefficient of $56.5 \mathrm{~cm}^{-1}$, and a gain-length product $g \times l=$ 18.4 .

delays that maximize the laser energy on these lines are about 620 and 520 ps, respectively.

Gain measurements for Ti targets at the optimized incidence angle of $20^{\circ}$ were conducted using a $350-\mathrm{mJ}$ prepulse and a 1-J picosecond heating pulse by monitoring the variation of the laser line intensity as a function of target length. Fig. 12 shows the laser output at $32.6 \mathrm{~nm}$ as a function of plasma length. For short plasma lengths the intensity of the laser output is observed to increase exponentially, with a small-signal gain coefficient of $56.5 \mathrm{~cm}^{-1}$, until saturation is reached. The gain-length product reaches 18.4 for a $4-\mathrm{mm}$ target.

Operation of the 32.6-nm laser at a repetition rate of $5 \mathrm{~Hz}$ is illustrated in Fig. 13, for a series of 250 consecutive shots. Lasing is observed in all shots, while intensity variation is characterized by a standard deviation of $17 \%$ of the mean. The maximum soft X-ray laser pulse energy observed is estimated to be $780 \mathrm{~nJ}$, while the average energy of these 250 shots is $530 \mathrm{~nJ}$. This last number corresponds to an average output

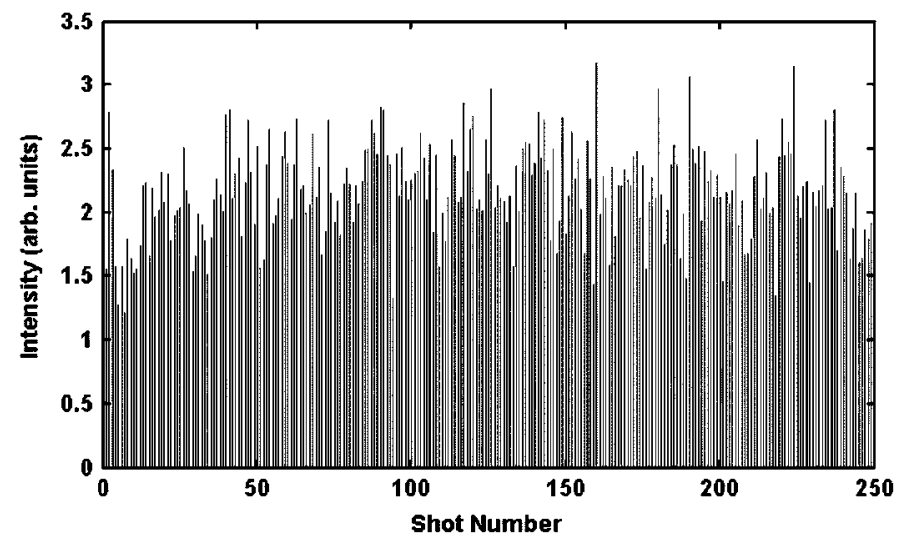

Fig. 13. Shot to shot variation of the intensity of the 32.6-nm laser line of $\mathrm{Ni}-\mathrm{like} \mathrm{Ti}$ at $5-\mathrm{Hz}$ repetition rate. The standard deviation of the series is $17 \%$ of the mean.

power of about $2.6 \mu \mathrm{W}$. This is to our knowledge the highest average power obtained from a soft X-ray laser operating in the vicinity of $30 \mathrm{~nm}$.

\section{GRAZING InCiDENCE PUMPING EXPERIMENTS With A 527-nm LASER BEAM}

This section describes the results of experiments conducted at LLNL using a 1054-nm long pulse beam in combination with a 527-nm short pulse beam. After initial experiments at the LLNL using a $10-\mathrm{Hz}, 800-\mathrm{nm}$ wavelength Ti:sapphire laser where Ni-like Mo lasing was demonstrated at $18.9 \mathrm{~nm}$ [17], [18], the grazing incidence experiments were transferred to the Compact Multipulse Terawatt (COMET) laser to study shorter wavelength Ni-like ion schemes at different pump wavelengths [12]. The smaller Ti:sapphire system at LLNL could produce a total of $150 \mathrm{~mJ}$ in the two beams required for pumping the GRIP scheme. This limited the range of laser pump energy, line focus parameters and target materials available for study. In particular, increasing the target line focus length and width was important to maximize X-ray laser output. We describe the experimental conditions using the small 4.2-cm-diameter beams of the COMET laser system where the $1 \omega, 1054-\mathrm{nm}$ long pulse beam was used in conjunction with the $2 \omega, 527-\mathrm{nm}$ short pulse beam.

The long pulse laser $1 \omega$, (1054 nm) 600-ps pulse with 1.2-1.3 J energy was focused to a line of 8-mm-long $\times 40-\mu \mathrm{m}$-wide (FWHM) using a spherical and cylindrical lens combination. The short pulse beam with approximately $2 \mathrm{~J}$ energy at 1054-nm wavelength compressed to $\sim 1.5$-ps duration was frequency doubled to $527 \mathrm{~nm}$ with $\sim 70 \%$ conversion efficiency. The $2 \omega$ beam was delivered to the target chamber by high-reflectivity, $527-\mathrm{nm}$ dielectric mirrors. The final $2 \omega$ short pulse energy was $1.3-1.5 \mathrm{~J}$ and was focused in a line of $\sim 7$-mm-long $\times 40-\mu$ m-wide (FWHM) with an on axis 15.4-cm-diameter parabola with focal length of 60.9 $\mathrm{cm}$. The angle of incidence of the short pulse beam to the target was chosen at $\theta=10^{\circ}$ which dictated the beam turning point at a plasma electron density of $1.2 \times 10^{20} \mathrm{~cm}^{-3}$. The Ni-like Pd ion and $\mathrm{Ag}$ ion $4 d-4 p$ lasers at 14.7 and $13.9 \mathrm{~nm}$, respectively, were chosen for this study. The targets consisted 


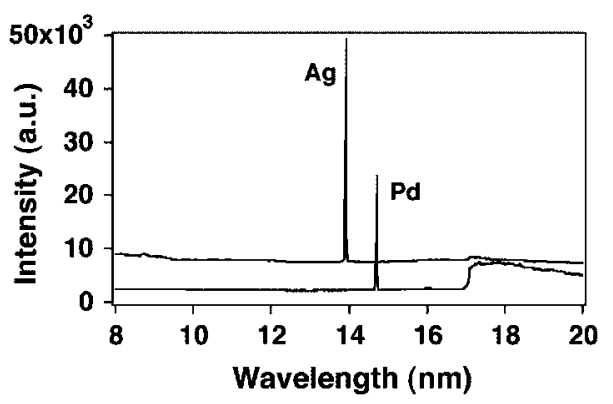

Fig. 14. Ni-like Pd and Ni-like Ag spectra from targets irradiated with a 1.3-J, 1054-nm, 600-ps pulse, and a 1.5-J, 527-nm, 1.5-ps short pulse. Both target lengths are $8 \mathrm{~mm}$. The Pd, Ag spectra are filtered with 200-nm Al and 200-nm Lexan/75 nm Al, respectively, to prevent the CCD detector from saturating.

of a 1-mm-thick Pd slab and a Mo slab coated with 3- $\mu \mathrm{m}$ Ag. A 1200 line/mm flat field grating instrument coupled to a $1024 \times 1024\left(24 \times 24 \mu \mathrm{m}^{2}\right.$ pixel $)$ back-illuminated CCD camera observed the soft $\mathrm{X}$-ray emission on axis from the target. After some initial optimization it was found that there was very strong output available in the X-ray laser with an optimum delay of 200 ps peak-to-peak between the two laser pump pulses. Fig. 14 shows the intense spectra from 8-mm-long $\mathrm{Ag}$ and $\mathrm{Pd}$ targets, measured through the peak of the X-ray laser lines. The spectra are offset vertically for clarity. The $\mathrm{X}$-ray laser output is sufficiently intense to require substantial attenuation using thin foil filters. The delay window was $\sim 200$ ps (FWHM). This window is wider by $3-4 \times$ than the previous Ni-like Mo GRIP results obtained with $150 \mathrm{~mJ}$ pump energy [18], but narrower than those observed in the Ni-like Pd results of Fig. 7 and in Ni-like Mo experiments that used $1 \mathrm{~J}$ of short pulse pump energy [19], [20]. The gain was determined to be $\sim 40 \mathrm{~cm}^{-1}$ for the Pd laser and was observed to go into saturation with $g L \sim 14$ at 4-mm target lengths with continued increase in output for targets up to $6 \mathrm{~mm}$. Longer targets up to $8 \mathrm{~mm}$ were irradiated giving higher X-ray laser output. The horizontal deflection angle of the $14.7-\mathrm{nm}$ X-ray laser as it leaves the plasma was slightly less than $5 \mathrm{mrad}$ away from the Pd target with a divergence of $\sim 9 \mathrm{mrad}$ (FWHM), Fig. 15(a). The deflection angle was very similar for the Ag X-ray laser with a divergence of $\sim 7 \mathrm{mrad}$ (FWHM), Fig. 15(b). Some variation in the beam divergence profiles was observed under different pumping conditions and will be discussed in a later publication. These results indicate that the GRIP X-ray laser output from a 6-mm Pd target are comparable with a $1.2-\mathrm{cm}$ target pumped transversely with $\sim 5 \mathrm{~J}$ in the short pulse. This indicates that a reduction of $3-4 \times$ in short pulse pump energy can be achieved for the same X-ray laser output.

\section{CONCLUSION}

We have discussed the physics and the implementation of compact high repetition rate soft X-ray lasers based on transient heating of laser created plasmas by a picosecond laser pulse impinging at grazing incidence. This excitation scheme takes advantage of the refraction of the pump beam in the plasma to significantly reduce the amount of laser pump energy required

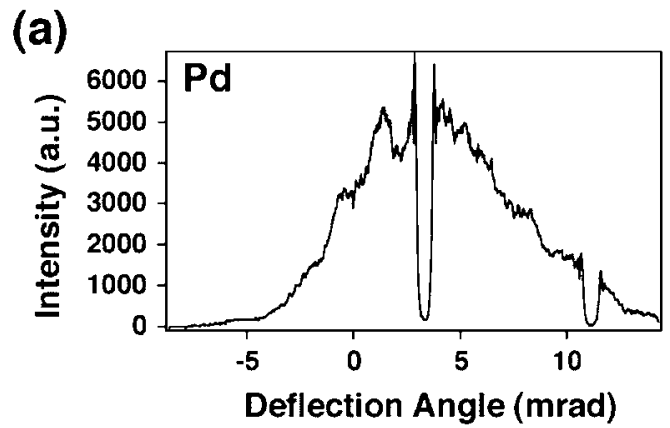

(b)

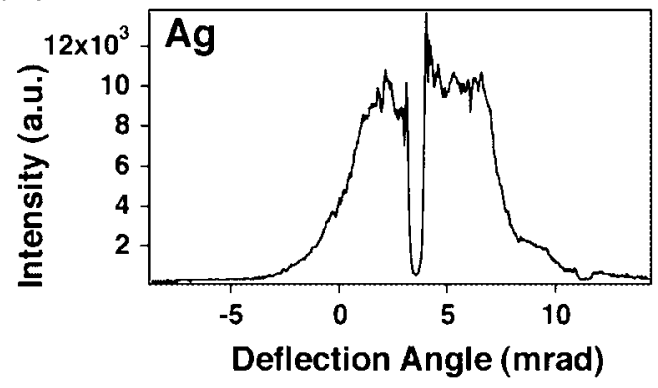

Fig. 15. Plots of the horizontal beam divergence and deflection angles for the (a) Ni-like Pd and (b) Ag spectral lines relative to the target surface. The dips in the profiles are from fiducial wires aligned between the target and the on axis spectrometer.

for the operation of collisional soft X-ray lasers in the gain-saturated regime. The generation of microwatt laser average powers from transitions of Ne-like and Ni-like ions in the 13.9-33-nm spectral region was demonstrated. The results show the feasibility of constructing high average power table-top soft X-ray for applications.

\section{REFERENCES}

[1] D. L. Matthews, P. L. Hagelstein, M. D. Rosen, M. J. Eckart, N. M. Ceglio, A. U. Hazi, H. Medecki, B. J. Macgowan, J. E. Trebes, B. L. Whitten, E. M. Campbell, C. W. Hatcher, A. M. Hawryluk, R. L. Kauffman, L. D. Pleasance, G. Rambach, J. H. Scofield, G. Stone, and T. A. Weaver, "Demonstration of a soft-X-ray amplifier," Phys. Rev. Lett., vol. 54, pp. 110-113, 1985.

[2] A. Carillon, H. Z. Chen, P. Dhez, L. Dwivedi, J. Jacoby, P. Jaegle, G. Jamelot, J. Zhang, M. H. Key, A. Kidd, A. Klisnick, R. Kodama, J. Krishnan, C. L. S. Lewis, D. Neely, P. Norreys, D. O’Neill, G. J. Pert, S. A. Ramsden, J. P. Raucourt, G. J. Tallents, and J. Uhomoibhi, "Saturated and near-diffraction-limited operation of an XUV laser at 23.6 nm," Phys. Rev. Lett., vol. 68, pp. 2917-2920, 1992.

[3] R. Smith R., G. J. Tallents, J. Zhang, G. Eker, S. McCabe, G. J. Pert, and E. Wolfrum, "Saturation behavior of two X-ray lasing transitions in Ni-like Dy," Phys. Rev. A, vol. 59, pp. R47-R50, 1999.

[4] J. J. Rocca, "Table top soft-X lasers," Rev. Sci. Instr, vol. 70, pp. 3799-3827, 1999.

[5] B. R. Benware, C. D. Macchieto, C. H. Moreno, and J. J. Rocca, "Demonstration of a high average power tabletop soft X-ray laser," Phys. Rev. Lett., vol. 81, pp. 5804-5806, 1998.

[6] J. J. Rocca, M. Frati, B. Benware, M. Seminario, J. Filevich, M. Marconi, K. Kanizay, A. Ozols, I. A. Artiukov, A. Vinogradov, and Y. A. Uspenskii, "Capillary discharge tabletop soft X-ray lasers reach new wavelengths and applications," C. R. Acad. Sci. Paris, vol. 1, pp. 1065-1081, 2000.

[7] G. Vaschenko, F. Brizuela, C. Brewer, M. Grisham, H. Mancini, C. S Menoni, M. Marconi, J. J. Rocca, W. Chao, A. Liddle, E. Anderson, D. Attwood, A. V. Vinogradov, I. A. Artioukov, Y. P. Pershyn, and V. V. Kondratenko, "Nano-imaging with a compact extreme ultraviolet laser," Opt. Lett., vol. 30, pp. 2095-2097, 2005. 
[8] M. G. Capeluto, G. Vaschenko, M. Grisham, M. C. Marconi, S. Luduena, L. Pietrasanta, Y. Lu, B. Parkinson, C. S. Menoni, and J. J. Rocca, "Nanopatterning with interferometric lithography using a compact $\lambda=46.9 \mathrm{~nm}$ laser," IEEE Trans. Nanotechnol., submitted for publication.

[9] S. Sebban, R. Haroutunian, P. Balcou, G. Grillon, A. Rousse, S. Kazamias, T. Marin, J. P. Rousseau, L. Notebaert, M. Pittman, J. P. Chambaret, A. Antonetti, D. Hulin, D. Ross, A. Klisnick, A. Carillon, P. Jaegle, G. Jamelot, and J. F. Wyart, "Saturated amplification of a collisionally pumped optical-field-ionization soft X-ray laser at $41.8 \mathrm{~nm}$," Phys. Rev. Lett., vol. 86, pp. 3004-3007, 2001.

[10] S. Sebban, T. Mocek, D. Ross, L. Upcraft, P. Balcou, R. Haroutunian, G. Grillon, B. Rus, A. Klisnick, A. Carillon, G. Jamelot, C. Valentin, A. Rousse, J. P. Rousseau, L. Notebaert, M. Pittman, and D. Hulin, "Demonstration of a Ni-like Kr optical-field-ionization collisional soft X-ray laser at 32.8 nm,” Phys. Rev. Lett., vol. 89, p. 253 901, 2002.

[11] P. V. Nickles, V. N. Shlyaptsev, M. Kalachnikov, M. Schnurer, I. Will, and W. Sandner, "Short pulse X-ray laser $32.6 \mathrm{~nm}$ based on transient gain in Ne-like titanium," Phys. Rev. Lett., vol. 78, pp. 2748-2751, 1997.

[12] J. Dunn, Y. Li, A. L. Osterheld, J. Nilsen, J. R. Hunter, and V. N. Shlyaptsev, "Gain saturation regime for laser-driven tabletop, transient Ni-like ion X-ray lasers,” Phys. Rev. Lett., vol. 84, pp. 4834-4837, 2000.

[13] R. Li and Z. Z. Xu, "Highly efficient transient collisional excitation X-ray laser in Ni-like Mo ions," in Proc. 7th Int. Conf. X-Ray Lasers, vol. 11, G. Jamelot, C. Möller, and A. Klishnick, Eds., Saint-Malo, France, 2001, pp. 27-34.

[14] T. Ozaki, R. A. Ganeev, A. Ishizawa, T. Kanai, and H. Kuroda, "Highly directive $18.9 \mathrm{~nm}$ nickel-like molybdenum X-ray laser operating at 150 mJ pump energy," Phys. Rev. Lett., vol. 89, p. 253 902, 2002.

[15] R. Tommasini, J. Nilsen, and E. E. Fill, "Investigations on 10-Hz subJoule fs-laser pumped neon- and nickel-like X-ray lasers," Proc. SPIE, Soft X-Ray Lasers and Applications IV, vol. 4505, pp. 85-92, 2001.

[16] V. N. Shlyaptsev, J. Dunn, S. Moon, R. Smith, R. Keenan, J. Nilsen, K. B. Fournier, J. Kuba, A. L. Osterheld, J. J. Rocca, B. Luther, Y. Wang, and M. Marconi, "Numerical studies of transient and capillary X-ray lasers and their applications," Proc. SPIE, Soft X-Ray Lasers and Applications $V$,, vol. 5197, pp. 221-228, 2003.

[17] R. Keenan, J. Dunn, V. N. Shlyaptsev, R. Smith, P. K. Patel, and D. F. Price, "Efficient pumping schemes for high average brightness collisional X-ray lasers," Proc. SPIE, Soft X-Ray Lasers and Applications $V$, vol. 5197, pp. 213-220, 2003.

[18] R. Keenan, J. Dunn, P. K. Patel, D. F. Price, R. F. Smith, and V. N. Shlyaptsev, "High repetition rate grazing incidence pumped X-ray laser operating at 18.9 nm," Phys. Rev. Lett., vol. 94, p. 103 901, 2005.

[19] B. M. Luther, Y. Wang, M. Larotonda, D. Alessi, M. Berrill, M. Marconi, V. Shlyaptsev, and J. J. Rocca, "Saturated high repetition rate 18.9 nm table-top laser in nickel-like molybdenum," Opt. Lett., vol. 30, pp. 165-167, 2005.

[20] M. A. Larotonda, B. M. Luther, Y. Wang, Y. Liu, D. Alessi, M. Berrill, A. Dummer, F. Brizuela, C. S. Menoni, M. C. Marconi, V. N. Shlyaptsev, J. Dunn, and J. J. Rocca, "Characteristics of a saturated 18.9-nm tabletop laser operating at 5-Hz repetition rate," IEEE J. Sel. Topics Quantum Electron., vol. 10, no. 6, pp. 1363-1367, Nov./Dec. 2004.

[21] Y. Wang, M. A. Larotonda, B. M. Luther, M. C. Marconi, D. Alessi, M. Berrill, V. N. Shlyaptsev, and J. J. Rocca, "Demonstration of saturated high repetition rate tabletop soft X-ray lasers at wavelengths down to 13.9 nm," Phys. Rev. A., vol. 72, p. 053807, 2005.

[22] D. Alessi, B. M. Luther, Y. Wang, M. A. Larotonda, M. Berrill, and J. J. Rocca, "High repetition rate operation of saturated table-top soft X-ray lasers in transitions of neon-like ions near $30 \mathrm{~nm}$," Opt. Expr., vol. 13, pp. 2093-2098, 2005.

[23] R. A. London, "Beam optics of exploding foil plasma X-ray lasers," Phys. Fluids, vol. 31, pp. 184-192, 1988.

[24] G. J. Tallents et al., "Saturated and short pulse duration X-ray lasers," Proc. AIP 8th Int. Conf. X-Ray Laser, vol. C641, pp. 291-297, 2002.

[25] J. Nilsen, "Analysis of a picosecond-laser-driven Ne-like Ti X-ray laser," Phys. Rev. A, vol. 5, pp. 3271-3274, 1997.

Bradley M. Luther received the B.S. in chemistry from Texas A\&M University, College Station, and the Ph.D. degree in physical chemistry from Colorado State University, Fort Collins.

He is currently a Research Scientist at the NSF Center for Extreme UV Science and Technology.
Yong Wang received the B.S. degree in precise instrument engineering from Tianjin University, Tianjin, China, in 1994, and the M.S. degree from Tianjin Physics and Chemistry Institute, Tianjin, in 1997. He is currently working toward the Ph.D. degree at Colorado State Univesity, Fort Collins, working on the $\mathrm{X}$-ray laser research and development.

Miguel A. Larotonda received the Licenciatura degree in physics and the Doctorado in physics in 2002 from Buenos Aires University, Buenos Aires, Argentina, working at the Centro de Investigaciones en Laseres y Aplicaciones (CITEFA-CONICET) where he was focused on studying the dynamics of short pulse all-solid-state lasers.

After receiving his Doctorado, he spent one year at the Quantum Electronics Laboratory, Universidad de Buenos Aires, working on a two-color pump and probe experiment to study lifetimes of excited states on wide bandgap crystals. At present, he is a Posdoctoral Fellow at the Electrical Engineering Department, Colorado State University, Fort Collins. His present research interests include the development of new laser sources in the extreme-UV region of the spectrum and their use in applications, as well as the development of high-peak power visible laser systems.

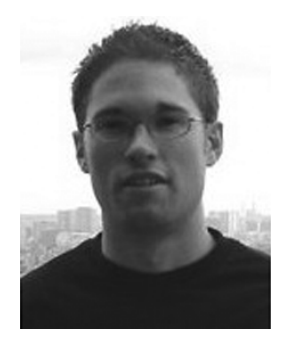

David Alessi (S'99) was born in 1981 in Durango, CO. He received the B.S. (hons.) degree in electrical and computer engineering from Colorado State University, Fort Collins, in 2003. He is currently working toward the M.S. degree in electrical and computer engineering.

His research interests include the development of high average power EUV lasers, solid state lasers and plasmas.

Mr. Alessi is a student member of SPIE and Eta Kappa Nu.

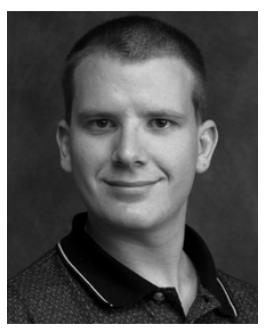

Mark Berrill (S'99) received the B.S. degree in electrical and computer engineering from Colorado State University, Fort Collins, in 2003, where he is currently working toward the Ph.D. degree in electrical and computer engineering.

His researh interests are in plasma modeling and extreme ultraviolet lasers.

Mr. Berrill is a member of SPIE, Sigma Xi, and Eta Kappa Nu.

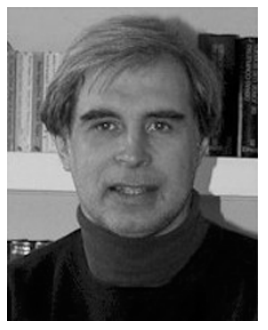

Jorge J. Rocca (SM'80-M'83-SM'94-F'00) is a Professor of electrical and computer engineering and physics at Colorado State University, Fort Collins.

He serves as Director of the NSF Center for Extreme Ultraviolet Science and Technology. His current research interests are in the development and physics of compact extreme ultraviolet and soft $\mathrm{X}$-ray lasers, and the applications of EUV light. $\mathrm{He}$ has conducted research and published more than 100 papers in peer review journals on topics related to short wavelength radiation sources, lasers, and plasmas. He and collaborators pioneered the development of table-top discharge pumped soft X-ray lasers and their use in numerous applications, including the interferometry of dense plasmas. His group is also currently involved in the development and study of high repetition rate laser-pumped soft X-ray lasers.

Prof. Rocca served as Associate Editor of the IEEE JouRnAL OF QUANTUM ELECTRONICS for 1994-1999, as Guest Editor of the IEEE JOURNAL OF SELECTED TOPICS IN QUANTUM ELECTRONICS, and as a member of the Editorial Board of the Review of Scientific Instruments. He was a National Science Foundation Presidential Young Investigator for 1985-1990. He is a Fellow of the Optical Society of America and a member of the American Physical Society. 
James Dunn received the Ph.D. degree in high resolution X-ray spectroscopy of laboratory sources at the University of Leicester, Leicester, U.K., in 1990.

He worked at the National Research Council of Canada, Ottawa, ON, as a Postdoctoral Researcher and studied laser-driven X-ray lasers, atomic spectroscopy of highly charged ions, and the characterization of laser-produced plasmas. He joined the Lawrence Livermore National Laboratory (LLNL), Livermore, CA, in 1992 to study high-power, short-pulse laser matter interaction physics. He started working on tabletop laser-driven X-ray lasers at LLNL in 1997 and continues to develop ultrabright X-ray sources for different applications. His other research interests include the physics of hot, dense, highly ionized plasmas.

Dr. Dunn is a member of the American Physical Society and the Optical Society of America.

R. Keenan, photograph and biography not available at the time of publication.
Vyacheslav N. Shlyaptsev received the Ph.D. degree in physics and mathematics from P.N. Lebedev Physics Institute, Moscow, Russia, in 1987.

He was with P.N. Lebedev Physics Institute from 1982 to 1993, and worked on the theory and modeling of X-ray lasers with A. V. Vinogradov and plasma hydrodynamics with Yu. V. Afanasiev. In 1993-1996, he conducted research in the Electrical Engineering Department, Colorado State University, Fort Collins, together with the group of J. J. Rocca on capillary discharge X-ray lasers. Since 1997, he has been a Research Associate in the Department of Applied Sciences, University of California at Davis-Livermore. He conducts research in laser produced and z-pinch plasmas numerical modeling, X-ray lasers, and ICF. 